\title{
Genome-Wide Characterization of Glutathione Peroxidase (GPX) Gene Family in Rapeseed (Brassica napus L.) Revealed Their Role in Multiple Abiotic Stress Response and Hormone Signaling
}

\author{
Wei Li ${ }^{1, *,+}$, Xuemin Huai ${ }^{1,+}$, Peitao Li ${ }^{1,+}$, Ali Raza ${ }^{2, *(D)}$, Muhammad Salman Mubarik ${ }^{3}\left(\mathbb{D}\right.$, Madiha Habib $^{3} \mathbb{D}$, \\ Sajid Fiaz ${ }^{4}$, Binbin Zhang ${ }^{1}$, Jun Pan ${ }^{1}$ and Rao Sohail Ahmad Khan ${ }^{3, *}$ (D) \\ 1 Beijing Goldenway Bio-Tech Co., Ltd., Chaoyang District, Beijing 100015, China; \\ huaixuemin@jiabowen.com (X.H.); lipeitao@jiabowen.com (P.L.); zhangbinbin@jiabowen.com (B.Z.); \\ panjun@jiabowen.com (J.P.) \\ 2 Fujian Provincial Key Laboratory of Crop Molecular and Cell Biology, Oil Crops Research Institute, \\ Center of Legume Crop Genetics and Systems Biology, College of Agriculture, Fujian Agriculture and \\ Forestry University (FAFU), Fuzhou 350002, China \\ 3 Centre of Agricultural Biochemistry and Biotechnology (CABB), University of Agriculture, \\ Faisalabad 38040, Pakistan; msmubarik@gmail.com (M.S.M.); madihahabib217@gmail.com (M.H.) \\ check for \\ updates \\ Citation: Li, W.; Huai, X.; Li, P.; Raza, \\ A.; Mubarik, M.S.; Habib, M.; Fiaz, S.; \\ 4 Department of Plant Breeding and Genetics, The University of Haripur, Haripur 22600, Pakistan; \\ sfiaz@uoh.edu.pk \\ * Correspondence: liwei@jiabowen.com (W.L.); alirazamughal143@gmail.com (A.R.); \\ srao@uaf.edu.pk (R.S.A.K.) \\ + These authors equally contributed to this work.
} Zhang, B.; Pan, J.; Khan, R.S.A. Genome-Wide Characterization of Glutathione Peroxidase (GPX) Gene Family in Rapeseed (Brassica napus L.) Revealed Their Role in Multiple Abiotic Stress Response and Hormone Signaling. Antioxidants 2021, 10, 1481. https://doi.org/ 10.3390/antiox10091481

Academic Editors: Fernanda Fidalgo, Anket Sharma and Cristiano Soares

Received: 23 August 2021

Accepted: 15 September 2021

Published: 17 September 2021

Publisher's Note: MDPI stays neutral with regard to jurisdictional claims in published maps and institutional affiliations.

Copyright: (C) 2021 by the authors Licensee MDPI, Basel, Switzerland. This article is an open access article distributed under the terms and conditions of the Creative Commons Attribution (CC BY) license (https:/ / creativecommons.org/licenses/by/ $4.0 /)$.

Abstract: Plant glutathione peroxidases (GPXs) are the main enzymes in the antioxidant defense system that sustain $\mathrm{H}_{2} \mathrm{O}_{2}$ homeostasis and normalize plant reaction to abiotic stress conditions. To understand the major roles of the GPX gene family in rapeseed (Brassica napus L.), for the first time, a genome-wide study identified 25 BnGPX genes in the rapeseed genome. The phylogenetic analysis discovered that GPX genes were grouped into four major groups (Group I-Group IV) from rapeseed and three closely interrelated plant species. The universal investigation uncovered that the $B n G P X s$ gene experienced segmental duplications and positive selection pressure. Gene structure and motifs examination recommended that most of the BnGPX genes demonstrated a comparatively wellmaintained exon-intron and motifs arrangement within the identical group. Likewise, we recognized five hormones-, four stress-, and numerous light-reactive cis-elements in the promoters of BnGPXs. Five putative bna-miRNAs from two families were also prophesied, targeting six BnGPXs genes. Gene ontology annotation results proved the main role of BnGPXs in antioxidant defense systems, ROS, and response to stress stimulus. Several BnGPXs genes revealed boosted expression profiles in many developmental tissues/organs, i.e., root, seed, leaf, stem, flower, and silique. The qRT-PCR based expression profiling exhibited that two genes (BnGPX21 and BnGPX23) were suggestively up-regulated against different hormones (ABA, IAA, and MeJA) and abiotic stress (salinity, cold, waterlogging, and drought) treatments. In short, our discoveries provide a basis for additional functional studies on the $B n G P X$ genes in future rapeseed breeding programs.

Keywords: antioxidant; abiotic stress; genomics; gene expression; gene ontology; hormones; miRNA; rapeseed breeding

\section{Introduction}

Plants are frequently exposed to several environmental stresses, comprising both abiotic and biotic, which significantly affect the growth and developmental processes [1-4]. These traumas can boost the production of reactive oxygen species (ROS), impairing cellular apparatuses and macromolecules, including DNA, proteins, and lipids, and eventually 
program the cells' death [5-7]. In plants, ROS are chemical species formed by an inadequate drop of oxygen; these species are well thought out as key signaling molecules, regulating stress responses and contributing to plant production [6]. Primarily, ROS are produced in the apoplast, mitochondria, plasma membrane, chloroplast, peroxisomes, endoplasmic reticulum, and cell walls [5,6]. Consequently, plants have advanced enzymatic and nonenzymatic antioxidative defense systems to cope with the harmfulness of ROS [5,6,8].

Among different antioxidant enzymes, glutathione peroxidase (GPX; EC 1.11.1.9) is an effective ROS scavenging enzyme that goes to the non-heme thiol peroxidase family and observes glutathione (GSH) and thioredoxin (Trx) as dipping substrata [9]. In comparison to mammals GPXs, plants GPXs have a partiality to Trx as a replacement for GSH as the dipping substratum $[10,11]$. Furthermore, plants' GPXs comprise cysteine (Cys) in their functioning sites, whereas usually, mammals' GPXs comprise selenocysteine (SeCys) residue as a substitute to Cys $[9,11,12]$.

In the recent past, several investigations have shown that the increased/up-regulated activity/expression of GPX enzyme and GPX genes help plants to cope with various environmental stresses. For instance, increased GPX activity helps different plants to improves tolerance to oxidative stress in cucumber (Cucumis sativus L.), tobacco (Nicotiana tabacum), and rice (Oryza sativa L.) seedlings [13]; water-deficit stress in wheat (Triticum aestivum L.) [14]; salicylic acid-induced salinity stress in tomato (Solanum lycopersicum L.) [15]; brassinosteroids-induced low-temperature stress tolerance in tomato [16]; etc. Similarly, the increased/up-regulated expression levels of GPX genes increased the tolerance to water-deficit stress in wheat [14]; drought tolerance in Hevea clones [17]; lead-induced oxidative stress tolerance in wheat [18]; etc. However, in some cases, the stressed plants also decreased the expression levels of GPX genes; for example, several GPX genes were down-regulated in response to drought stress in sorghum (Sorghum bicolor L.) [19]. The down-regulated expression of GPX genes could participate in the activation of some downstream mechanisms.

Some functional studies showed, for example, that the silencing of mitochondrial GPX1 gene in rice activated the damage of photosynthesis against salinity stress [20]. Knockdown of two GPX genes, including OsGPX1 and OsGPX3, could cruelly disturb the steady growth and developmental processes in rice [21]. Overexpression of Rhodiola crenulata RcGPX5 gene raises drought stress tolerance in Salvia miltiorrhiza plants [22]. In another investigation, the overexpression of the Arabidopsis AtGPXL5 gene resulted in changed plant growth and redox status under salinity stress in Arabidopsis [23]. In a nutshell, these studies specified that plant GPXs genes could regulate plant developmental processes, stress responses, tolerance mechanisms.

Rapeseed (Brassica napus L.) is the second most significant oilseed crop and holds a complex genome. Various abiotic stresses meaningfully restrict rapeseed production [24-27]. To our best knowledge, the GPX gene family has not been documented in rapeseed. Hence, for the first time, the current study executed a genome-wide analysis to recognize GPX genes in the rapeseed genome. To get further insight into GPX genes evolution in rapeseed, their phylogenetic relations, synteny investigation, gene structures, conserved motifs, ciselements, miRNA predictions, and functional annotation were categorized. Furthermore, their expression profile in several tissues/organs and under several hormones and abiotic stress environments were broadly evaluated, which profoundly boosted our knowledge of the GPX genes in rapeseed.

\section{Materials and Methods}

\subsection{Documentation of GPX Gene Family in Rapeseed}

As defined in recent reports [28,29], two methods were employed to classify GPX genes in the Brassica napus genome, including BLASTP and the Hidden Markov Model (HMM) [28]. The sequence of Brassica napus genome was downloaded from the BnPIR database (http:/ / cbi.hzau.edu.cn/bnapus/index.php, accessed on 1 July 2021) [30]. For BLASTP, we used eight Arabidopsis thaliana GPXs (Table S1) amino acid sequences as an 
enquiry by e-value set to $1 \mathrm{e}^{-5}$. The amino acid sequences of eight $A t G P X s$ were retrieved from the TAIR Arabidopsis genome database (http:/ / www.arabidopsis.org/, accessed on 1 July 2021) [31]. Moreover, a local software, HMMER 3.1 [32], was used for hunting the GPX genes with default constraints. Then, the HMM profile of the GPX domain (Pfam: PF00255) obtained as of the Pfam database (http:/ / pfam.xfam.org/, accessed on 1 July 2021) was employed to detect GPX gene sequences. Finally, $25 B n G P X$ genes were recognized by merging the two methods in the rapeseed genome. Furthermore, we recognized GPX genes in diverse plant species, i.e., Brassica rapa and Brassica oleracea, and their genome sequences downloaded from the JGI Phytozome 12.0 database (https: / / phytozome.jgi.doe.gov / pz/ portal.html, accessed on 1 July 2021) [33] through a similar method.

\subsection{Characterization of GPX Gene Family}

The physico-chemical characteristics, including molecular weight and isoelectric points, were examined by means of the online ProtParam tool (http:/ / web.expasy.org/ protparam/, accessed on 1 July 2021) [34]. The subcellular localization of BnGPX proteins was prophesied from the WoLF PSORT server (https:/ / wolfpsort.hgc.jp/, accessed on 1 July 2021) [35]. The genes structures of BnGPX was created via TBtools software (V 1.068; https:/ / github.com/CJ-Chen/TBtools, accessed on 1 July 2021) [36]. The conserved motifs of BnGPX protein sequences were recognized by employing the MEME website (https:/ / meme-suite.org/meme/db/motifs, accessed on 1 July 2021) [37].

\subsection{Phylogenetic Tree and Synteny Analysis of BnGPX Family Proteins}

To perceive the evolutionary relationship of the $B n G P X$ gene family, we created a phylogenetic tree for Brassica napus, Brassica oleracea, Brassica rapa, and A. thaliana protein sequences. The sequence alignment was carried out using MEGA 7 software (https:// megasoftware.net/home, accessed on 1 July 2021) [38]. The neighbor-joining (NJ) scheme was executed to develop a phylogenetic tree with 1000 bootstrap replicates using Evolview v3 website (https: / / www.evolgenius.info/evolview, accessed on 1 July 2021) [39] to show the phylogenetic tree. Synteny relationships of GPX genes were established by pythonpackage, JCVI (https: / / github.com/tanghaibao/jcvi, accessed on 1 July 2021) [40] from Brassica napus, Brassica oleracea, Brassica rapa, and Arabidopsis thaliana. We precalculated the $\mathrm{Ka} /$ Ks ratios of all GPXs by employing KaKs_Calculator 2.0 software (https:/ / sourceforge. net/projects/kakscalculator2/, accessed on 1 July 2021) [41].

\subsection{Cis-Elements Analysis in the BnGPX Gene Promoters}

To study the putative cis-elements in the BnGPXs promoters, we obtained the $2 \mathrm{~Kb}$ sequence upstream of start codons in the Brassica napus genome. Then, the promoter sequence of the individual gene was examined using the PlantCARE website (http:/ / bioinformatics.psb.ugent.be/webtools/plantcare/html/, accessed on 1 July 2021) [42], and the figure was drawn using TBtools (V 1.068) [36].

\subsection{Estimation of Presumed miRNA Targeting BnGPX Genes and Functional Annotation Study}

The coding sequence (CDS) of BnGPXs was used to recognize possible target miRNAs in the psRNATarget database (http:/ / plantgrn.noble.org/psRNATarget/, accessed on 1 July 2021) [43] with default constraints. The interaction network between the miRNAs and BnGPX genes was drawn by Cytoscape software (V3.8.2) (https:/ / cytoscape.org/ download.html, accessed on 1 July 2021). Gene ontology (GO) annotation examination was accomplished by uploading all BnGPXs protein sequences to the eggNOG website (http:/ / eggnog-mapper.embl.de/, accessed on 1 July 2021) [44]. Then, TBtools was used to execute the $\mathrm{GO}$ enrichment examination.

\subsection{Expression Analysis of BnGPX Genes in Diverse Tissues}

For tissue-specific expression profiling, we downloaded RNA-seq data (BioProject ID: PRJCA001495, accessed on 1 July 2021) of rapeseed from the National Genomics Data 
Center. The complete method was described in a recent work [28]. Mainly, cuffquant and Cuffnorm were employed to create normalized counts in transcripts per million (TPM) values. Based on TPM estimates, the expression heat map was formed using GraphPad Prism 8 software (https:/ / www.graphpad.com/, accessed on 1 July 2021) [45].

\subsection{Plant Material and Stress Conditions}

In the current study, a typical cultivated variety, ZS11, was used for stress treatments. The seeds of the ZS11 genotype were obtained from Wuhan Zhongyou Seed Technology Co., Ltd., Wuhan, China. The stress treatments were performed as designated in previous work [28]. Briefly, the vigorous seeds were carefully chosen and sterilized with $10 \%$ hypochlorous acid solution for $5 \mathrm{~min}$. The seeds were grown on water-saturated filter paper in a chamber $\left(25^{\circ} \mathrm{C}\right.$ day/night and $16 \mathrm{~h} / 8 \mathrm{~h}$ light/dark cycle) until the radicle's extent turned up about $5 \mathrm{~mm}$. For stress treatment, sprouted seeds were uncovered to $150 \mathrm{mM} \mathrm{NaCl}$ solution for salt stress, 15\% PEG6000 solution for drought stress, and $4{ }^{\circ} \mathrm{C}$ for cold stress on water-saturated filter paper. For waterlogging stress, the seeds were flooded with water in the Eppendorf tube. To explore the influence of numerous hormones, the germinated seeds were planted in Murashige and Skoog (MS) medium supplied with $100 \mu \mathrm{M}$ of each hormone such as abscisic acid (ABA), gibberellic acid (GA), methyl jasmonate (MeJA), and indole-acetic acid/auxin (IAA). The samples were collected at 0 (CK), 2, 4, 6, and $8 \mathrm{~h}$ after the treatments. Three biological repetitions were carried out for all the treatments. All the samples were rapidly frozen in liquid nitrogen and were kept at $-80{ }^{\circ} \mathrm{C}$ for expression analysis.

\subsection{RNA Extraction and qRT-PCR Analysis}

Total RNA extraction and cDNA synthesis was performed using TransZol Up Plus RNA Kit (TransGen Biotech, Beijing, China) and cDNA Synthesis SuperMix (TransGen Biotech, Beijing, China) according to manufacturer instructions. The qRT-PCR was conducted with an ABI StepOne real-time fluorescence quantitative PCR instrument (Applied Biosystems, Foster City, CA, USA) using SYBR ${ }^{\circledR}$ Green Supermix (Bio-Rad). The BnACTIN was used as an internal control. The qRT-PCR reaction was performed as follows: $94{ }^{\circ} \mathrm{C}$ for $10 \mathrm{~min}$, followed by 40 cycles of $94{ }^{\circ} \mathrm{C}$ for $15 \mathrm{~s}, 60^{\circ} \mathrm{C}$ for $30 \mathrm{~s}$, and $72{ }^{\circ} \mathrm{C}$ for $10 \mathrm{~s}$. Each qRT-PCR reaction was carried out with three biological triplicates, and the data were observed using the $2^{-\Delta \Delta C T}$ method $[28,46]$. All the primers used in this experiment are listed in Table S1. The heatmap was formed using GraphPad Prism 8 software [45].

\section{Results}

\subsection{Documentation and Characterization of GPX Gene Family in Rapeseed}

Based on BLASTP examinations against the rapeseed genome employing eight Arabidopsis AtGPXs proteins as enquiries, a total of 25 presumed GPX genes were recognized in the complete rapeseed genome (Table 1). Henceforth, these genes are named "BnGPX1$B n G P X 25$ ", among which 13 genes (BnGPX1-BnGPX13) were located on the A subgenome, and 12 genes (BnGPX14-BnGPX25) were located on the C subgenome (Table 1). Comprehensive data of the $25 B n G P X$ genes is shown in Table 1 . In brief, gene length varied from 1028 bp (BnGPX17) to 4654 bp (BnGPX9) with 5-6 exons in each sequence (Table 1). Eleven genes comprise six exons, and 14 genes consist of five exons (Table 1$)$. The CDS length varied from $363 \mathrm{bp}$ ( $B n G P X 10)$ to $726 \mathrm{bp}$ (BnGPX23), whereas the protein length altered from 120 (BnGPX10) to 241 (BnGPX23) amino acids (Table 1). The predicted molecular weights of the $25 \mathrm{BnGPX}$ proteins ranged from $13.45 \mathrm{kDa}$ (BnGPX10) to $26.97 \mathrm{kDa}$ (BnGPX22), and the isoelectric points ranged from 5.18 (BnGPX24) to 9.58 (BnGPX22) (Table 1). The subcellular localization results anticipated that $16 \mathrm{BnGPX}$ proteins are located on the chloroplast, 6 proteins are located on the cytoplasm, 2 proteins (BnGPX3 and BnGPX9) are located on extracellular, and 1 protein (BnGPX20) is located on the nucleus (Table 1). Moreover, 8 GPX genes (BolGPX1-BolGPX8) from the Brassica oleracea and 12 GPX genes were also discovered from Brassica rapa (BraGPX1-BraGPX12) genomes (Table S2). 
Table 1. Information of the $25 \mathrm{BnGPX}$ genes discovered in rapeseed genome.

\begin{tabular}{|c|c|c|c|c|c|c|c|c|c|}
\hline Gene ID & $\begin{array}{l}\text { Gene } \\
\text { Name }\end{array}$ & Gene Position (bp) & $\begin{array}{c}\text { Gene } \\
\text { Length } \\
\text { (bp) }\end{array}$ & $\begin{array}{c}\text { CDS } \\
\text { Length } \\
\text { (bp) }\end{array}$ & Exon & $\begin{array}{l}\text { Protein } \\
\text { Length } \\
\text { (AA) }\end{array}$ & $\begin{array}{l}\text { Molecular } \\
\text { Weight } \\
\text { (kDa) }\end{array}$ & $\begin{array}{l}\text { Isoelectric } \\
\text { Point (pI) }\end{array}$ & $\begin{array}{l}\text { Subcellular } \\
\text { Localization }\end{array}$ \\
\hline BnaA02T0266400ZS & $B n G P X 1$ & A02:18048650-18049870 - & 1220 & 690 & 6 & 229 & 25.28 & 9.21 & Chloroplast \\
\hline BnaA03T0152400ZS & $B n G P X 2$ & A03:7777692-7779631 - & 1939 & 510 & 6 & 169 & 18.92 & 6.34 & Cytoplasm \\
\hline BnaA03T0210900ZS & $B n G P X 3$ & A03:11008433-11009656 - & 1223 & 501 & 5 & 166 & 18.39 & 8.92 & Extracellular \\
\hline BnaA03T0529000ZS & $B n G P X 4$ & A03:29805209-29806435 + & 1226 & 720 & 5 & 239 & 26.84 & 9.46 & Chloroplast \\
\hline BnaA04T0003000ZS & BnGPX5 & A04:177901-178974 - & 1073 & 507 & 5 & 168 & 18.85 & 8.82 & Chloroplast \\
\hline BnaA04T0301400ZS & $B n G P X 6$ & A04:25656674-25657708 - & 1034 & 507 & 5 & 168 & 18.99 & 8.99 & Chloroplast \\
\hline BnaA05T0000100ZS & $B n G P X 7$ & A05:5755-6787 - & 1032 & 561 & 5 & 186 & 21.00 & 8.86 & Cytoplasm \\
\hline BnaA05T0124600ZS & $B n G P X 8$ & A05:7501774-7503157 + & 1383 & 510 & 6 & 169 & 18.93 & 6.34 & Cytoplasm \\
\hline BnaA07T0222500ZS & $B n G P X 9$ & A07:22717658-22722312 + & 4654 & 717 & 6 & 238 & 26.86 & 9.68 & Extracellular \\
\hline BnaA08T0269900ZS & BnGPX10 & A08:25981327-25984256 - & 2929 & 363 & 5 & 120 & 13.45 & 6.28 & Chloroplast \\
\hline BnaA09T0247900ZS & BnGPX11 & A09:18151781-18152884 - & 1103 & 525 & 5 & 174 & 19.09 & 6.81 & Chloroplast \\
\hline BnaA09T0577200ZS & BnGPX12 & A09:58245269-58246443 - & 1174 & 717 & 5 & 238 & 26.45 & 9.23 & Chloroplast \\
\hline BnaA09T0721600ZS & BnGPX13 & A09:65850924-65853242 + & 2318 & 513 & 6 & 170 & 19.20 & 9.17 & Chloroplast \\
\hline BnaC02T0362600ZS & BnGPX14 & C02:37887177-37888540 - & 1363 & 690 & 6 & 229 & 25.36 & 9.3 & Chloroplast \\
\hline BnaC03T0178400ZS & BnGPX15 & C03:10008604-10010540 - & 1936 & 510 & 6 & 169 & 18.99 & 5.6 & Cytoplasm \\
\hline BnaC03T0248900ZS & BnGPX16 & C03:15285876-15287196 - & 1320 & 630 & 5 & 209 & 23.29 & 8.76 & Chloroplast \\
\hline BnaC04T0001100ZS & BnGPX17 & C04:225671-226699+ & 1028 & 537 & 5 & 178 & 20.16 & 9.13 & Cytoplasm \\
\hline BnaC04T0156100ZS & $B n G P X 18$ & C04:14243676-14245017 + & 1341 & 510 & 6 & 169 & 18.93 & 6.34 & Cytoplasm \\
\hline BnaC04T0257400ZS & BnGPX19 & C04:36128046-36129247 - & 1201 & 549 & 6 & 182 & 20.30 & 8.58 & Chloroplast \\
\hline BnaC04T0617900ZS & BnGPX20 & C04:71165478-71166588+ & 1110 & 477 & 6 & 158 & 18.10 & 9.05 & Nucleus \\
\hline BnaC06T0239400ZS & $B n G P X 21$ & C06:34932165-34933231 + & 1066 & 540 & 5 & 179 & 20.22 & 9.18 & Chloroplast \\
\hline BnaC07T0506700ZS & $B n G P X 22$ & C07:58481304-58482530+ & 1226 & 720 & 5 & 239 & 26.97 & 9.58 & Chloroplast \\
\hline BnaC08T0428600ZS & $B n G P X 23$ & C08:47326442-47327687 - & 1245 & 726 & 5 & 241 & 26.71 & 9.33 & Chloroplast \\
\hline BnaC09T0141600ZS & $B n G P X 24$ & C09:10653827-10654957 + & 1130 & 561 & 5 & 186 & 21.09 & 5.18 & Chloroplast \\
\hline BnaC09T0292200ZS & $B n G P X 25$ & C09:28600420-28601680 - & 1260 & 516 & 6 & 171 & 18.80 & 8.32 & Chloroplast \\
\hline
\end{tabular}

In the genomic position, the positive $(+)$ and negative $(-)$ signs indicate the existence of a gene on the positive and negative strand of that specific marker, respectively.

\subsection{Phylogenetic Relationships of GPX Genes}

To discover the evolutionary history between the BnGPX, BolGPX, BraGPX, and AtGPX genes, an unrooted phylogenetic tree was assembled by a multiple sequence alignment (MSA) of the prophesied GPX protein sequences from Brassica napus, Brassica oleracea, Brassica rapa, and Arabidopsis thaliana, which was gathered into four main groups (Group I-Group IV) (Figure 1). The findings revealed that Group I was comprised of 10 GPX members (4 BnGPXs, 2 BraGPXs, 2 BolGPXs, and 2 AtGPX); Group II was comprised of 17 GPX members (7 BnGPXs, 4 BraGPXs, 3 BolGPXs, and 3 AtGPX); Group III contained 17 GPX members (9 BnGPXs, 2 BolGPX, 4 BraGPXs, and 2 AtGPXs); and Group IV was comprised of 9 GPX members (5 BnGPXs, 1 BolGPXs, 2 BraGPXs, and 1 AtGPXs) (Figure 1). Overall, GPXs clustering into the identical sub-group may possess parallel functions. It is worth mentioning that BnGPX genes were spread in each group with homologs from other plant species, and Group II and III were observed to have more BnGPXs, BolGPXs, $B r a G P X s$, and AtGPXs members than the other three groups (Figure 1). Additionally, it was noticed that the BnGPXs have a stronger phylogenetic link with the BolGPXs and BraGPXs in each group. 


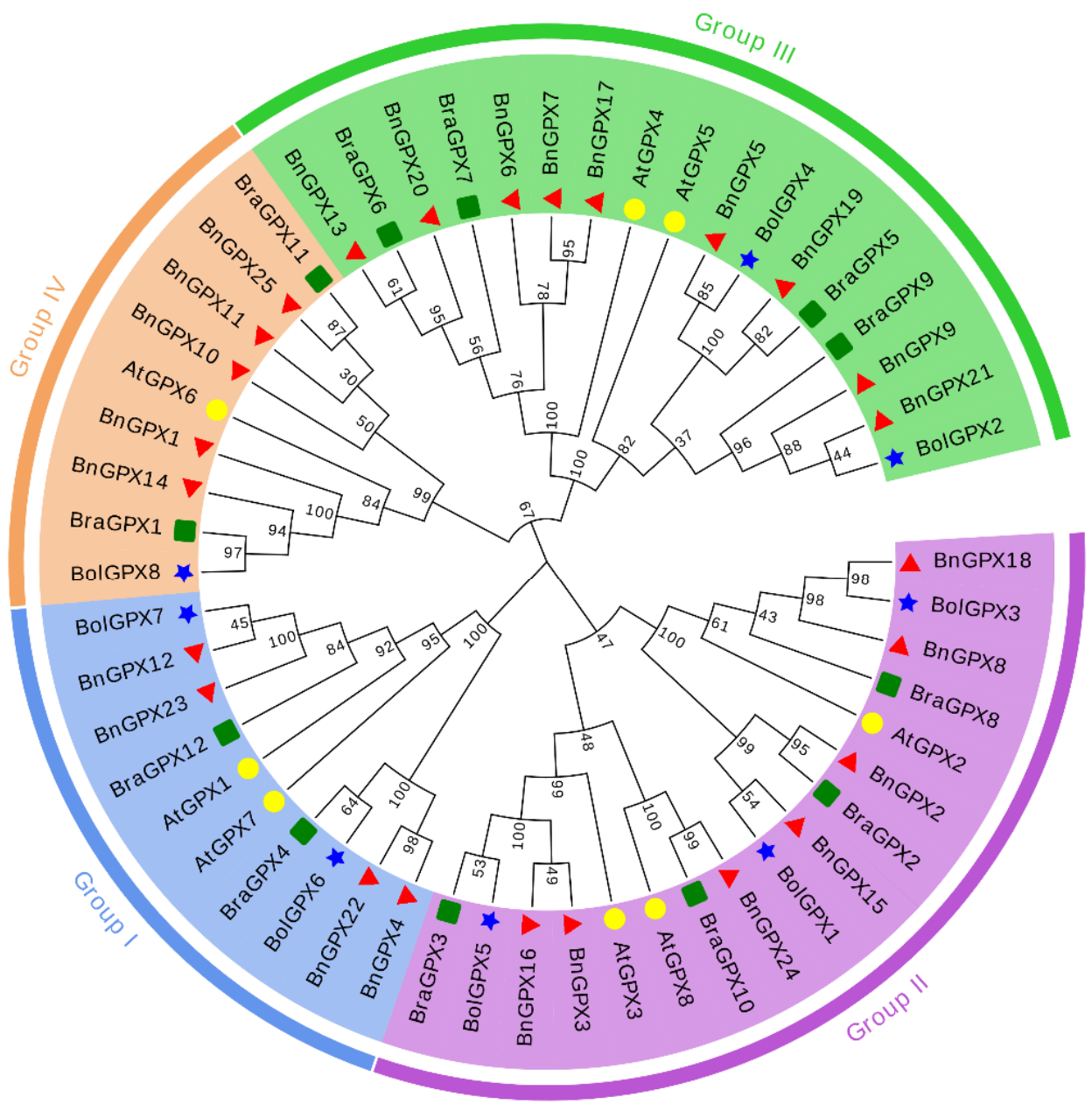

Figure 1. A neighbor-joining phylogenetic tree assessment of GPX genes in Brassica napus, Brassica oleracea, Brassica rapa, and Arabidopsis thaliana. Overall, 25 BnGPXs from Brassica napus (red triangle), 8 BolGPXs from Brassica oleracea (blue star), 12 BraGPXs from Brassica rapa (green box), and 8 AtGPXs from Arabidopsis thaliana (yellow circles) were clustered into four groups (Groups I-IV), symbolized by unique colors.

\subsection{Evaluation of Chromosomal Dispersal and Synteny Links of BnGPX Genes}

The development of new gene family followers in plants' genome advancement is comparatively ascribed to tandem and segmental doubling events [47]. Thus, the corresponding events were explored in BnGPXs to clarify the rapeseed GPX gene duplication events. The chromosomal scatterings of 25 BnGPX genes were appraised. Notably, 8 out of the 19 chromosomes had BnGPX genes (Figure 2). Concisely, chromosomes A03, A05, A07, $\mathrm{A} 09, \mathrm{C} 03, \mathrm{C} 04, \mathrm{C} 06$, and C08 had only one BnGPX gene (Figure 2). Remarkably, the residual chromosomes did not have any BnGPX gene, and no tandem repeat paralogous genes were found in any regions. Moreover, eight paralogous genes were identified on the A03, A05, A07, A09, C03, C04, C06, and C08 chromosomes (Figure 2). These conclusions revealed that the duplication events played a critical role in developing the BnGPX family genes. 


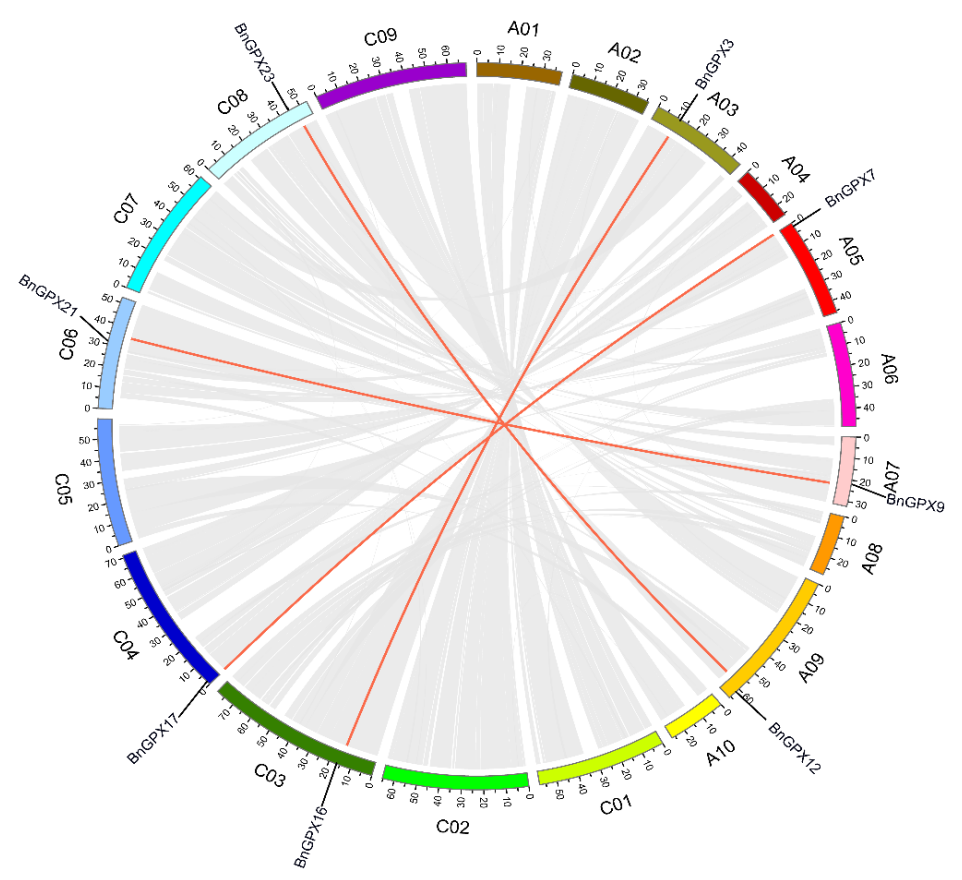

Figure 2. Circular drawings of the chromosomal distribution and inter-chromosomal organization of $B n G P X$ genes. Gray lines in the background signify all the syntenic blocks in the rapeseed genome and the red lines indicate syntenic GPX gene pairs.

Collinearity analysis uncovered robust orthologs of the GPX genes among Brassica napus and the other hereditary plant species (Figure 3; Table S3). In short, in the A subgenome, 7 Brassica napus genes exhibited syntenic links with 5 AtGPXs, 1 BraGPXs, and BolGPXs. In the $\mathrm{C}$ subgenome, 9 Brassica napus genes presented syntenic links with 4 AtGPXs, 3 BraGPXs, and 2 BolGPXs (Figure 3). Particularly, numerous homologues of Arabidopsis thaliana, Brassica rapa, and Brassica oleracea withstood a syntenic alliance with $B n G P X s$, implying that whole-genome duplication (segmental duplication) events portrayed a vital role in BnGPXs gene family evolution in the rapeseed genome (Table S3).

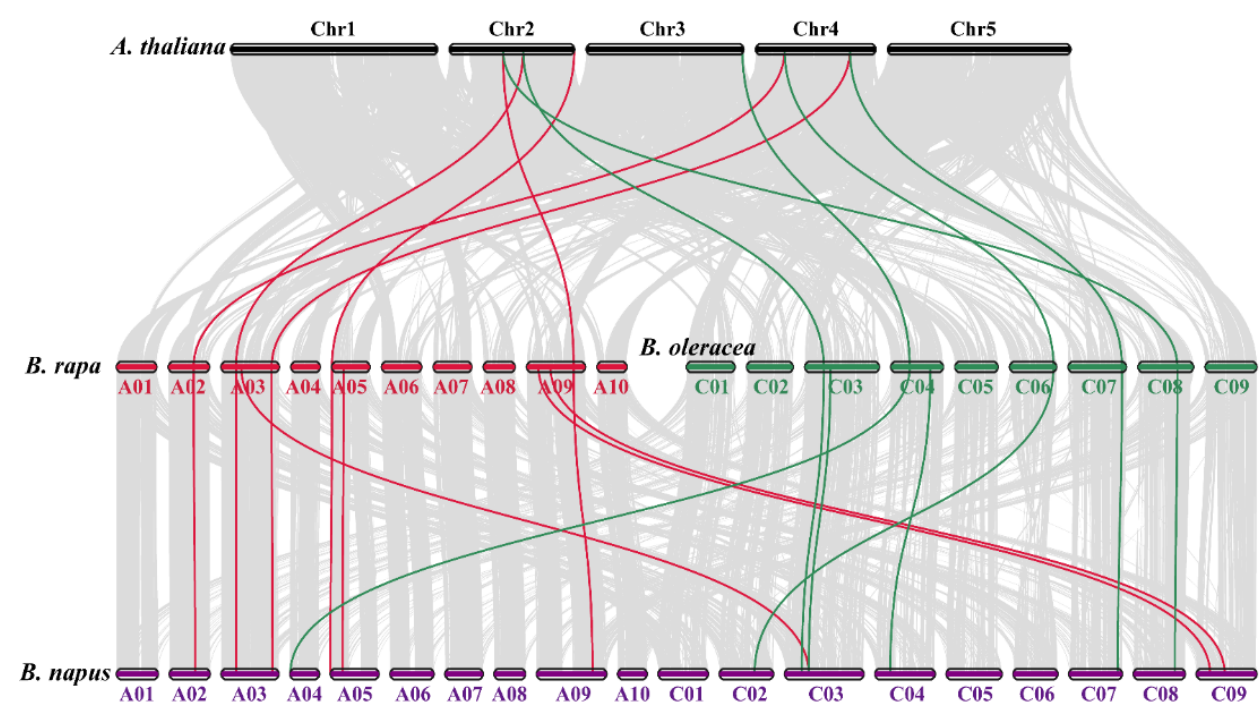

Figure 3. Synteny analysis of GPX genes in Brassica napus, Brassica rapa, Brassica oleracea, and Arabidopsis thaliana chromosomes. Gray lines in the background indicate the collinear blocks within Brassica napus and other plant genomes, whilst the red and green lines emphasize the syntenic GPX gene pairs. Genes located on the Brassica napus A and C sub-genomes are syntenic with Brassica rapa, Brassica oleracea, and Arabidopsis thaliana. 
The $\mathrm{Ka} / \mathrm{Ks}$ ratio is a substantial index in estimating the replication events and selection pressures [48]. Thus, to comprehend the evolutionary constraints on the BnGPX gene family, the $\mathrm{Ka}, \mathrm{Ks}$, and $\mathrm{Ka} / \mathrm{Ks}$ ratio was evaluated. The results stated that all the repeated $B n G P X$ gene pairs had a $\mathrm{Ka} / \mathrm{Ks}$ ratio of $<1$ (Table S3); however, two gene pairs (BnGPX4/BraGPX4 and $\mathrm{BnGPX7/BraGPX7)}$ had a $\mathrm{Ka} / \mathrm{Ks}$ ratio of $>1$ and experienced positive selection pressure (Table S3). Almost all genes experienced segmental duplication events. Similarly, Brassica rapa, Brassica oleracea, and Arabidopsis thaliana also experienced the purifying selective pressure (Table S3). Overall, the findings signify that the rapeseed GPX family genes might have encountered purifying and positive selection burden during the course of their evolution.

\subsection{Evaluation of Gene Structures and Conserved Motifs of BnGPX Genes}

The gene structures (exon-intron arrangements) of the BnGPX genes were examined to gain insight into the expansion of rapeseed GPX family genes. The results showed that introns of the BnGPXs ranged from 4-5 (Figure 4A), and exons ranged from 5-6 in each sequence (Table 1). Group I contained 4 introns, whereas Groups II-IV include 4-5 introns. A similar trend was also observed for exons (5-6/gene) within the same groups (Figure 4A; Table 1). Mainly, BnGPX2 and BnGPX15 have diverse gene structures in Group II. In short, Groups II, III, and IV exhibited analogous intron-exon arrangements, and only Group I had a distinct intron-exon arrangement. These outcomes stipulated that GPX members within a group astonishingly had the same gene structures, steady with their phylogenetic relatives.
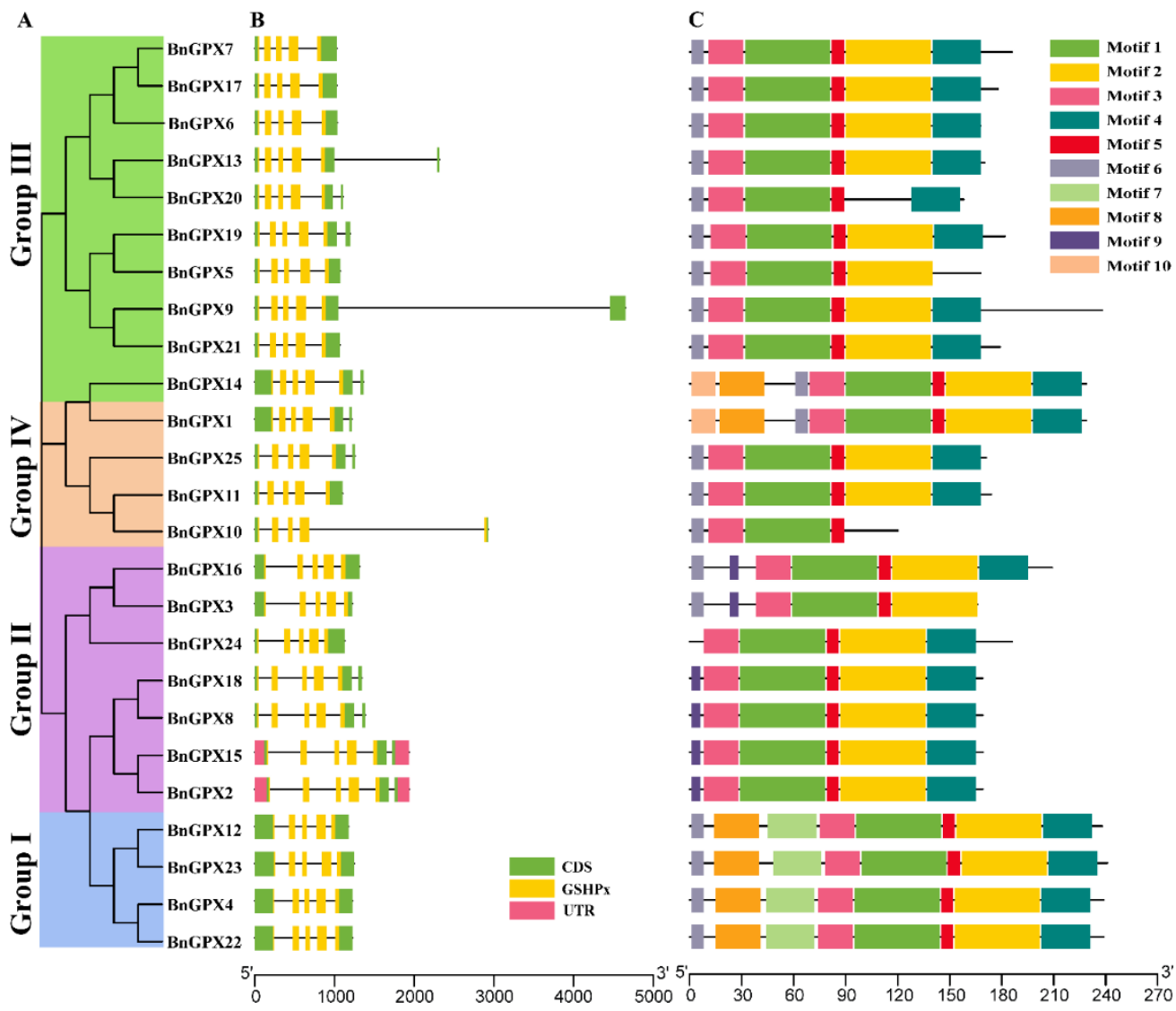

Figure 4. The gene structure and motif analysis of the GPX family genes from rapeseed. (A) Based on the phylogenetic interactions, the BnGPX genes are grouped into four groups (Groups I-IV). (B) The gene structure of the BnGPXs. Light green color indicates the CDS regions, pink color displays the UTR regions, yellow color displays the GSHPx domains, and black horizontal line demonstrates the introns. (C) Conserved motifs configurations are named in the BnGPXs. Unique color boxes demonstrate distinct motifs. 
Additionally, we examined the full-length protein sequences of 25 BnGPXs to distinguish their conserved motifs. The conserved motif of the BnGPX genes varied from 4-8. Overall, ten conserved motifs were recognized, and the motif dispersals were also analogous within the groups (Groups I-IV) (Figure 4B; Table S4). For example, all members of Group I comprise 8; Group II contains 5-7; Group III includes 5-8; and Group IV encompasses 4-8 conserved motifs (Figure 4B). Notably, only BnGPX10 had four motifs in Group IV (Figure 4B). Interestingly, motif 7 was specific to Group I; motif 9 was specific to Group II; motif 10 was limited to two genes (BnGPX14 and BnGPX1) in Groups III and IV; and other motifs were nearly equally distributed on all the genes (Figure 4B). In a nutshell, the group organization's evenness was strongly retained by exploring the conserved motifs dispersals, gene structures, and phylogenetic links, demonstrating that the GPX proteins have exceptionally preserved amino acid residues and members contained by a group might have analogous roles.

\subsection{Cis-Elements in the Promoters of BnGPX Genes}

To discriminate the gene functions and regulatory roles, cis-regulatory elements in $B n G P X$ s promoter regions were inspected by searching a 2000 bp upstream region from each gene's transcriptional activation site against the PlantCARE database. The complete data of cis-elements are offered in Table S5. The results display that five phytohormone-correlated (auxin, (abscisic acid (ABA), gibberellin (GA), methyl jasmonate (MeJA), and salicylic acid (SA)) responsive elements comprising TCA-element, TGA-element, CGTCA-motif, ABRE, AuxRR-core, TGACG-motif, TATC-box, GARE-motif, P-box, etc., were documented (Figure 5; Table S5). Predominantly, many phytohormone-connected elements were prophesied to be definite to some genes and extensively scattered (Figure 5), suggesting the decisive role of these genes in phytohormone arbitration.

Additionally, four abiotic stress-accompanying (drought, low-temperature, anaerobic, and light) elements, comprising ARE, LAMP-element, LTR, MRE, TCCC-motif, GT1-motif, chs-CMA1a, AE-box, box 4, G-box, MBS, etc. were recognized (Figure 5; Table S5). Primarily, several light-responsive elements were found to be extensively disseminated among all of the genes (Figure 5; Table S5), suggesting the extensive role of BnGPXs against light stress. On the whole, outcomes directed that BnGPXs expression levels may depart under phytohormone and abiotic stress environments.

\subsection{Genome-Wide Investigation of miRNA Targeting BnGPX Genes}

Earlier reports demonstrated that miRNA-mediated regulation is associated with the stress responses in plants. Hence, to enhance our familiarity of miRNA-mediated posttranscriptional regulation on $B n G P X s$, the current study discovered five miRNAs targeting six BnGPX genes (Figure 6A; Table S6). The miRNA-targeted sites are shown in Figure 6B, and the comprehensive data of all miRNAs targeted sites/genes are introduced in Table S6. The outcomes disclosed that 4 members of the bna-miR164 family targeted four genes ( $B n G P X 3, B n G P X 4, B n G P X 16$, and BnGPX22); and one member of the bna-miR396 family targeted two genes (BnGPX13 and BnGPX20) (Figure 6; Table S6). Primarily, BnGPX3, $B n G P X 4, B n G P X 16$, and BnGPX22 were projected to be targeted by a superior number of miRNAs (Figure 6A; Table S6). The expression profiling of predicted miRNAs and their targeted sites/genes needs confirmation in the further investigation to oversee their biological functions in the rapeseed genome. 

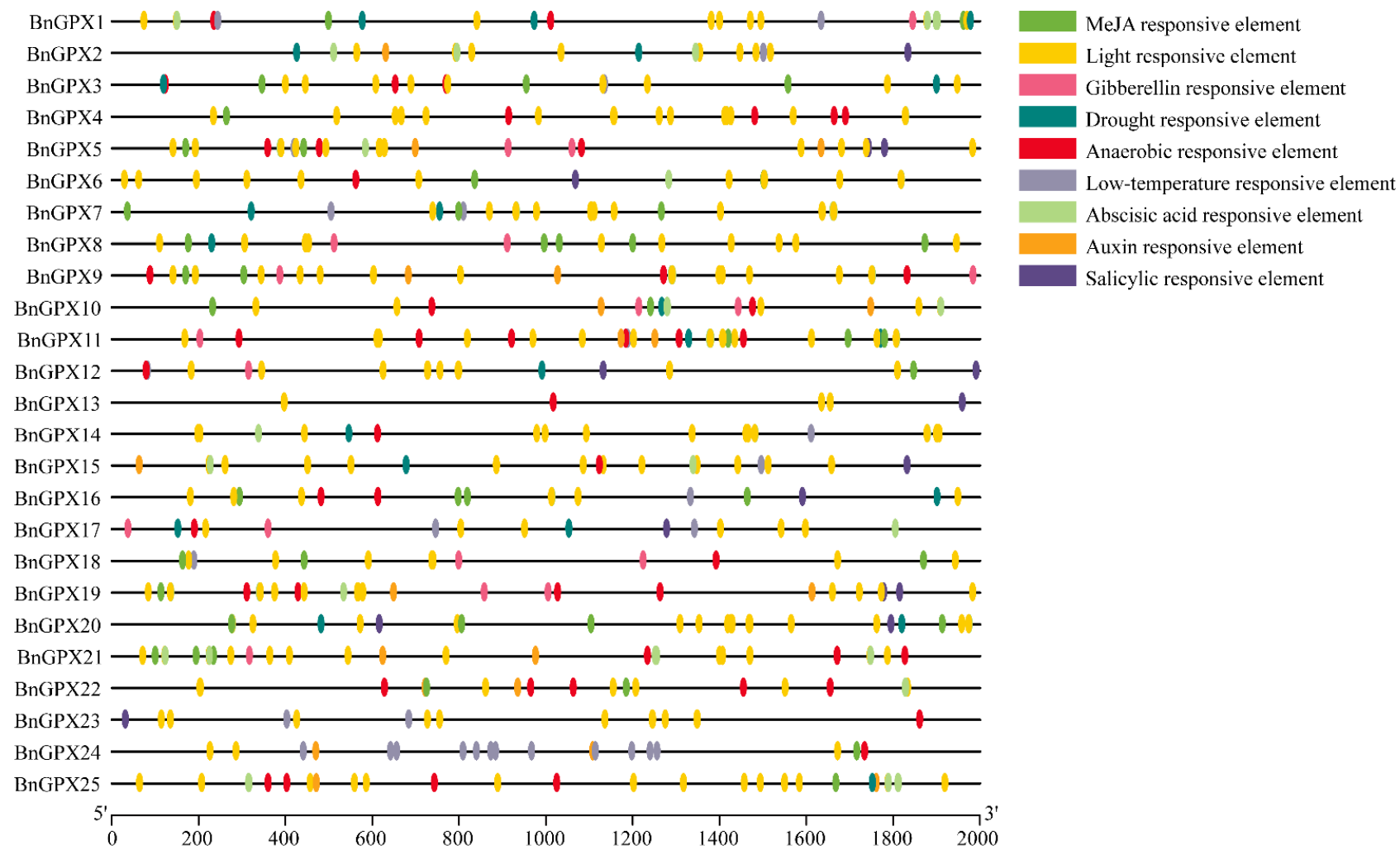

Figure 5. Evaluation of cis-regulatory elements in the BnGPXs promoters' regions that are linked with various hormoneand stress-responsive elements. Unique color boxes display unique identified cis-elements.

\subsection{Functional Annotation Evaluation of BnGPX Genes}

To further distinguish the BnGPX genes' roles, $\mathrm{GO}$ annotation and enrichment analysis were carried out based on biological process (BP), molecular function (MF), and cellular component (CC) classes. The BP, MF, and CC annotation conclusions presented abundant suggestively enriched terms (Table S7). For instance, the BP enrichment analysis uncovered that these genes were principally involved in response to hydrogen peroxide (GO:0042542), response to an organic substance (GO:0010033), cellular response to abiotic stimulus (GO:0071214), response to reactive oxygen species (GO:0000302), etc. (Table S7). Results of MF annotation revealed that these genes are involved in oxidoreductase activity (GO:0016684), antioxidant activity (GO:0016209), molecular_function (GO:0003674), glutathione peroxidase activity (GO:0004602), peroxidase activity (GO:0004601), etc. (Table S7). These terms also validate the function of BnGPX genes in ROS scavenging and antioxidant defense systems. The CC enrichment analysis confirms that these genes are mainly linked with cellular anatomical entity (GO:0110165), cytoplasm (GO:0005737), obsolete intracellular part (GO:0044424), obsolete cell part (GO:0044464), and organelle (GO:0043226), etc. (Table S7). Notably, few of these terms follow the prediction of subcellular localization of the GPX proteins. In short, CC, BP, and MF-GO annotation results proved the main role of $B n G P X s$ in antioxidant defense systems, ROS, and response to stress stimulus. 

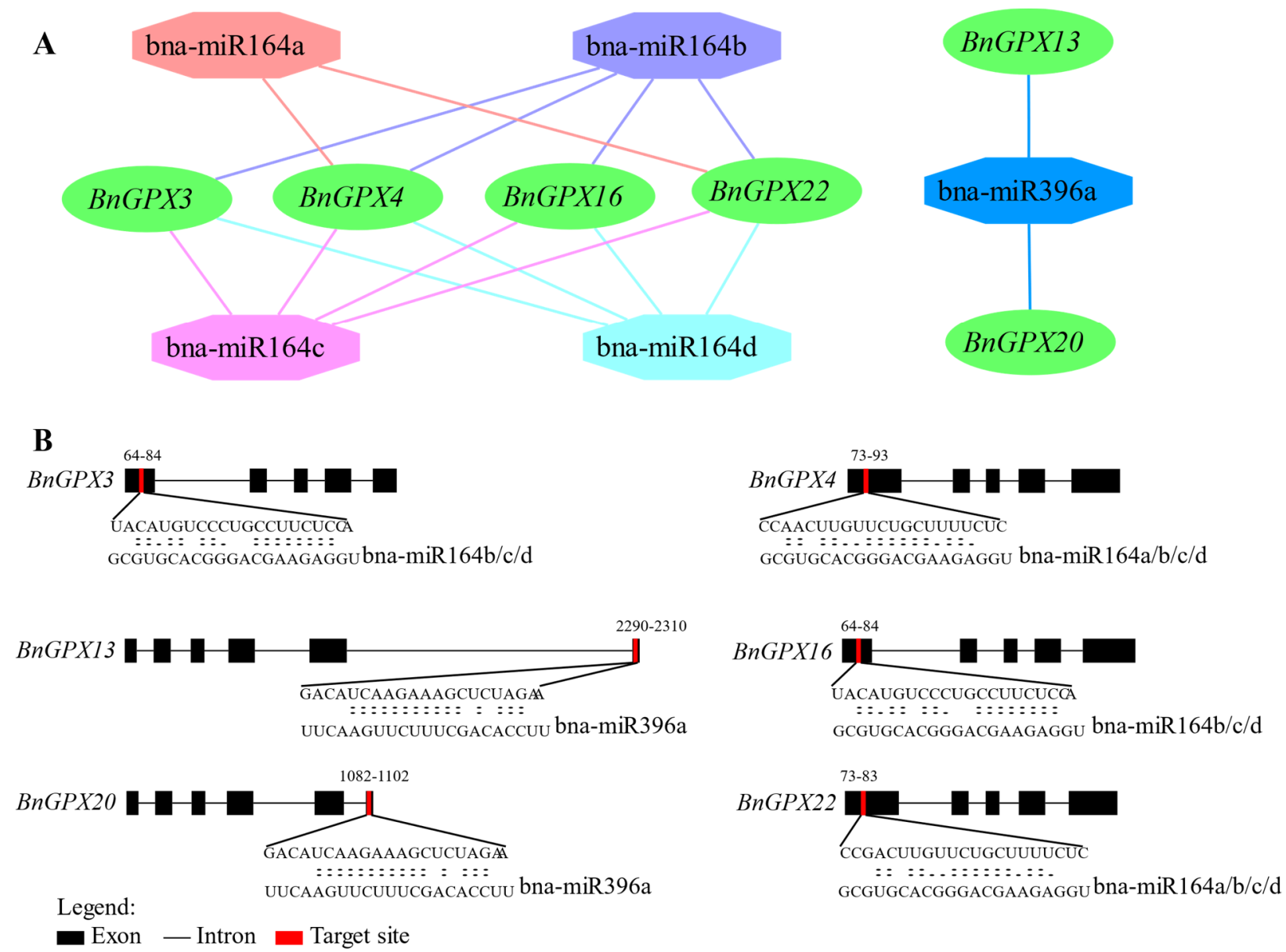

Figure 6. miRNA targeting $B n G P X$ genes. (A) Network diagram of predicted miRNA targeting $B n G P X$ genes. The green ellipse colors represent $B n G P X s$ genes, and different color shapes signify miRNAs. (B) The graphic illustration signifies the $B n G P X s$ gene targeted by miRNAs. The RNA sequence of each complementary site from $5^{\prime}-3^{\prime}$ and the predicted miRNA sequence from $3^{\prime}-5^{\prime}$ are visible in the long-drawn-out areas. See Table S6 for the comprehensive data of all predicted miRNAs.

\subsection{Tissue-Specific Expression Profiles of BnGPX Genes in Rapeseed}

The tissue-specific expression profiles of $B n G P X s$ genes were observed in six diverse tissues and organs, i.e., roots, stems, leaves, flowers, seeds, and silique, using RNA-seq data from Brassica napus (ZhongShuang 11 variety) (BioProject ID PRJCA001495). The expression profiles of the BnGPX genes altered in the different tissues and organs. As illustrated in Figure 7, group II genes exhibit higher expression in all of the tissues except $B n G P X 8, B n G P X 12$, and BnGPX18, which displayed relatively lower expression in seeds (Figure 7). On the other hand, group I and III genes show relatively lower expression in all of the tissues except $B n G P X 2, B n G P X 4, B n G P X 15$, and $B n G P X 22$ that showed somehow higher expression in leaf, flower, seeds, and silique (Figure 7). Thus, it is noteworthy that group II genes may play substantial roles in rapeseed developmental processes. 


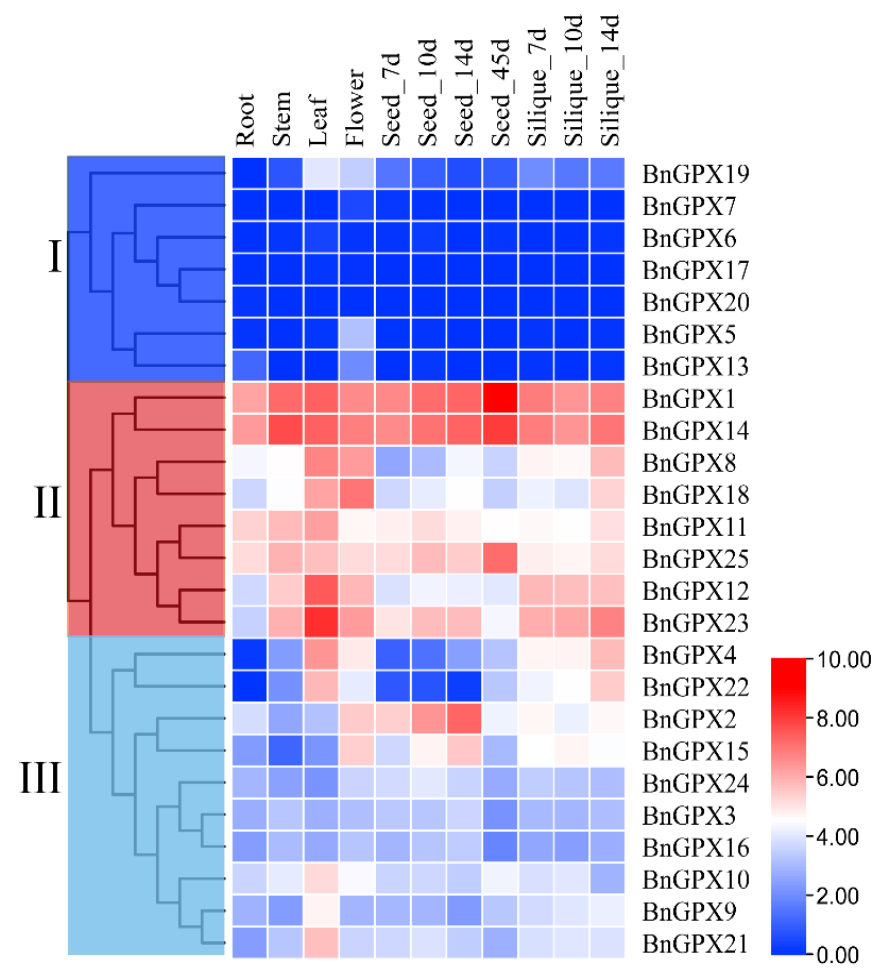

Figure 7. Expression profiles of BnGPX genes in numerous tissues at different growth stages of rapeseed. The $7 \mathrm{~d}, 10 \mathrm{~d}, 14 \mathrm{~d}$, and $45 \mathrm{~d}$ tags indicated the time-points when the samples were harvested. The red color indicates high and the blue color indicates low expression levels. The expression heat map was generated based on transcripts per million (TPM) values.

\subsection{Response of BnGPX Genes to Different Hormones and Abiotic Stress Treatments}

To investigate the BnGPX genes' expression profiles under various hormones (ABA, GA, IAA, and MeJA) and abiotic stress (salinity, cold, waterlogging, and drought) conditions, the qRT-PCR-based expression profiling of 8 randomly chosen $B n G P X$ genes was performed (Figure 8). In response to all of these stress environments, BnGPX21 and $B n G P X 23$ were up-regulated under IAA $(5.68,5.64,5.33$, and 3.68 folds for BnGPX21; $4.13,4.91,5.49$, and 5.22 folds for BnGPX23), salinity $(4.83,6.14,6.54$, and 5.81 folds for BnGPX21; 5.28, 5.40, 7.11, and 7.96 folds for BnGPX23), cold (4.09, 5.38, 4.5, and 3.21 folds for BnGPX21; 3.1, 3.9, 4.6, and 4.14 folds for BnGPX23), waterlogging (5.44, 6.09, 5.14, and 5.97 folds for BnGPX21; 1.84, 7.24, 6.51, and 4.70 folds for BnGPX23), and drought (5.71, $6.51,7.15$, and 7.59 folds for BnGPX21; 2.63, 9.09, 11.37, 14.10 folds for BnGPX23) conditions at $2,4,6$, and $8 \mathrm{~h}$, respectively, compared to CK (Figure 8), whereas BnGPX23 was also up-regulated under ABA $(5.55,7.27,9.43$, and 7.39 folds) and MeJA $(14.97,16.01,26.26$, and 26.03 folds) treatments at 2, 4,6, and $8 \mathrm{~h}$, respectively, compared to CK (Figure 8 ). Under GA treatment, only BnGPX2 was up-regulated $(10.9,11.04$, and 14.75 folds) at 2, 6, and $8 \mathrm{~h}$, respectively, compared to CK. On the other hand, BnGPX1 and BnGPX2 showed considerable expression under ABA, IAA, salinity, waterlogging, and drought conditions at certain time points (Figure 8). In comparison, the rest of the genes showed lower expression under all of the stress conditions. The results signify that mainly BnGPX21 and BnGPX23 genes could play a vital role in alleviating the harmful impact of different hormones and abiotic stress conditions in rapeseed. 

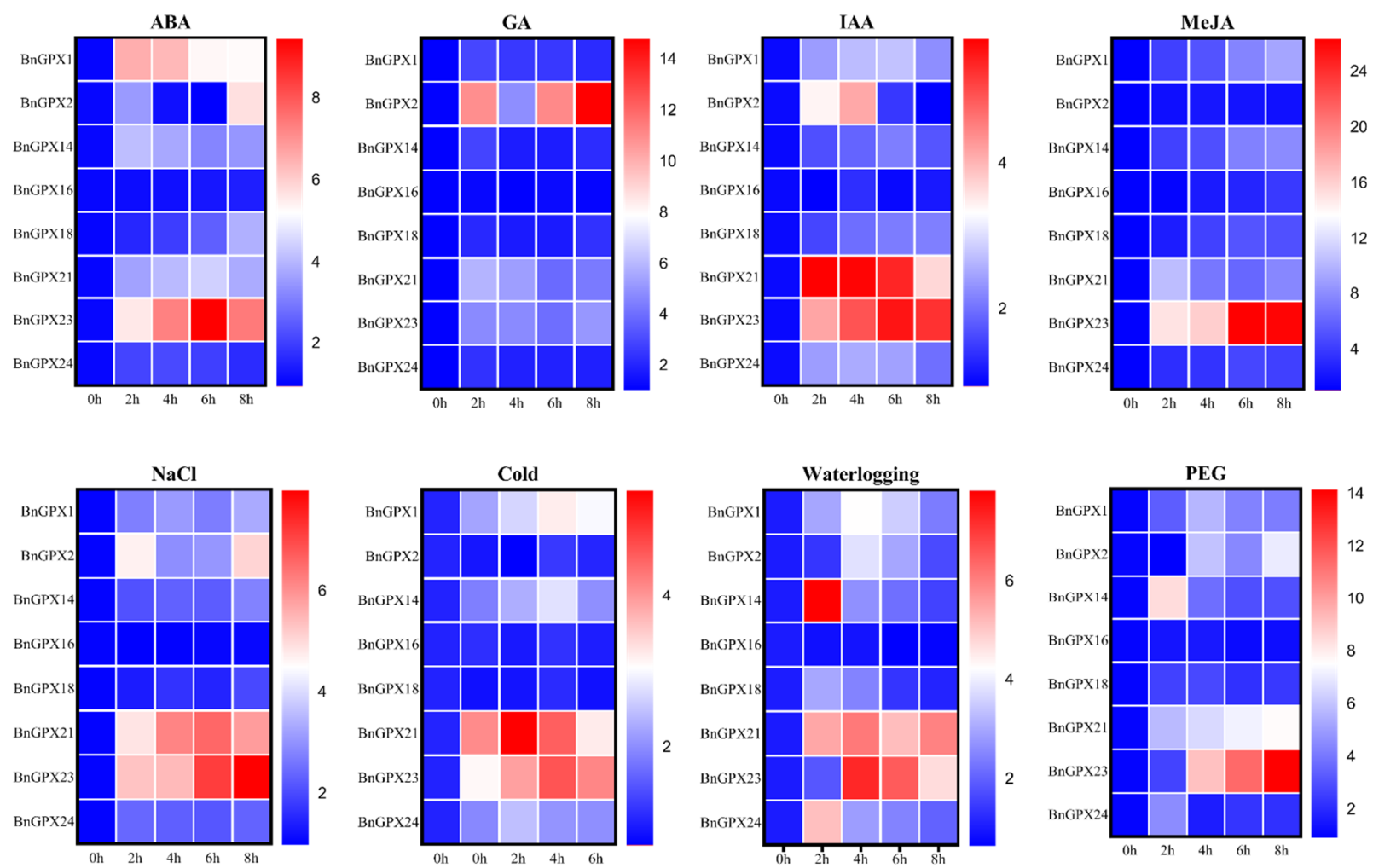

Figure 8. Expression profiles of BnGPX genes expression against various hormone and abiotic stress conditions at different time points $(0(\mathrm{CK}), 2,4,6$, and $8 \mathrm{~h}$ ). The red color shows high and the blue color shows low expression levels. The expression bars present the comparative gene expressions based on the $2^{-\Delta \Delta C T}$ method.

\section{Discussion}

Rapeseed is an allotetraploid crop that practices widespread genome replication and integration activities [49]. However, rapeseed yield is influenced by numerous abiotic pressures, including temperature (cold/heat), high salinity, drought, waterlogging, and heavy metals toxicity $[24,27,50]$. The generation of ROS in response to normal and stress atmospheres is assessed and scavenged via GPXs, which is considered one of the most effective ROS antioxidant scavenging enzymes [6,9]. Further, GPX enzyme and GPX genes also contribute to several plant physiological, biochemical, and molecular mechanisms to withstand harsh environmental stresses [5,6]. In the recent past, GPX gene families have been reported in numerous crop plants, including five GPX genes in date palm (Phoenix dactylifera L.) [51] and in Rhodiola crenulate [52]; six genes in cucumber (Cucumis sativus L.) [53], watermelon (Citrullus lanatus L.) [54], and Lotus japonicus [55]; seven genes in sorghum (Sorghum bicolor L.) [19]; eight genes in Thellungiella salsuginea [56] and peach fruit (Prunus persica L.) [57]; 13 genes in cotton (Gossypium hirsutum L.) [58]; and 14 genes in bread wheat [59]. To the best of our knowledge, the GPX gene family was yet to be reported in rapeseed that responds to multiple stress conditions. The availability of the entire rapeseed genome justifies the genome-wide description of the GPX gene family, which could be used for forthcoming rapeseed breeding programs.

According to the available literature, the current study identified the largest GPX gene family (25 BnGPXs) in the rapeseed genome (Table 1), which were grouped into four major groups (Figure 1). Deviations in the GPXs gene numbers between different plant species may be attributed to gene replication experiences, involving tandem and segmental replications, and participate in spreading GPXs for deviation. Gene replication of GPX genes was also uncovered in different plant species $[19,57,58]$. The current study showed that BnGPXs underwent segmental duplication, purifying and positive selection burden 
during their evolution (Table S3). Subsequently, these consequences recommended that $B n G P X s$ replication activities might play an imperative role in gene evolution.

The phylogenetic tree showed that GPXs genes from rapeseed and other three plant species, including Brassica rapa, Brassica oleracea, and Arabidopsis thaliana, were categorized into four main groups (Figure 1), which was constant with the grouping in other plant species such as Thellungiella salsuginea [56], cotton [58], sorghum [19], and bread wheat [59]. Fascinatingly, each group comprised one or more GPXs genes from all plant species, and every $B n G P X$ was extremely correlated to its homologs in the other three plant species, particularly in Brassica rapa and Brassica oleracea, suggesting that the mutual ancestor was self-possessed of pairs of possible orthologs. Additionally, gene structure analysis discovered that most of the BnGPX genes owned five introns (Figure 4; Table 1); however, the number of exons ranged widely from four to five, and introns number varied from five to six in each group (Figure 4). Similar findings were also reported in cucumber, where all $G P X$ genes had four to five introns and four to five exons [53]. In cotton, six exons and five introns were found in 13 GPX genes [58]. The exon-intron gatherings' divergence was practiced by three essential methods (exon/intron-gain/loss, exonization/pseudoexonization, and insertion/deletion), and they are precisely supported to structural divergence [60]. Remarkably, the GPX genes in each group offered parallel exon-intron organization and conserved motifs (Figure 4), indicating that these genes may take part in the matching roles associated with multiple abiotic cues. Similar discoveries have also been described in Thellungiella salsuginea [56], cotton [58], sorghum [19], and bread wheat [59].

To better comprehend the role of the BnGPX genes in contradiction of some environmental factors, cis-elements in the promoter regions were predicted. The outcomes demonstrated that two major kinds of cis-elements were identified, i.e., stress- and phytohormoneresponsive (Figure 5; Table S5). Almost all recognized cis-elements were allied with phytohormones (ABA, MeJA, GA, SA, and auxin), and abiotic stress (drought, low-temperature, light, and anaerobic induction) (Table S5). The previous report suggests that cis-elements support plant stress responses [61]. Moreover, these gene functions were additionally deeprooted by the $\mathrm{GO}$ annotation investigation (Table S7). Our results are in agreement with previous reports, where GPX genes contributed to several stress responses [19,51,55,56,59]. These conclusions can increase our comprehension of BnGPX genes under varied environmental circumstances.

In recent years, ample miRNAs have been documented by genome-wide investigation in rapeseed to take part in various environmental issues [62-66]. None of the previously reported GPXs gene families in different plants has reported miRNA-targeting GPX genes. Therefore, for the first time, the current study discovered five miRNAs from two families (miR164 and miR396) targeting six BnGPX genes (Figure 6; Table S6). In a recent study, $V m$-milR37 subsidized in pathogenicity through regulating the VmGPX gene, which takes part in the oxidative stress response throughout Valsa mali infection [67]. Notably, there is no report on miRNAs targeting GPX genes in plants. However, the identified miRNAs have been documented in different plants and stress conditions. For instance, miR164 regulated the salinity stress tolerance mechanism in maize (Zea mays L.) [68]. In another study, miR164-targeted NAC genes undesirably regulated drought stress tolerance in rice plants [69]. Further, miR164 negatively regulated the resistance of wheat to stripe rust [70]. Similarly, another miRNA (miR396) arbitrated amendment in plant growth and salt stress responses in creeping bentgrass (Agrostis stolonifera) [71]. Further, miR396 was found to be elaborated in plant response to vernalization and flower advancement in Agrostis stolonifera [72]. In another study, the overexpression of osa-MIR396c declines salinity and alkali stress tolerance in rice [73]. It is worth mentioning that these findings support our outcomes and endorse that bna-miRNAs might play critical roles in the contradiction of various stresses by fluctuating the transcript profiles of GPX genes in rapeseed.

Growing evidence specifies that GPXs genes may display diverse expression profiles in different organs/tissues and under stress circumstances $[19,51,55,56,59]$. Hence, the tissuespecific expression profiles of $B n G P X s$ genes were evaluated in six diverse developmental 
tissues using RNA-seq data (Figure 7), and these results are in agreement with previous reports. For example, six watermelon ClGPX genes presented higher expressions in flowers and fruits [54]. In cotton, almost all GhGPXs genes exhibited the uppermost expression in flowers [58]. In cucumber, some CsGPX genes showed higher expression in roots and flowers [53]. The innumerable expression profiles of BnGPX genes (mainly from Group II) specify that they may play diverse purposeful roles at certain developmental procedures in rapeseed.

Similarly, the expression profiles of eight BnGPX genes were appraised under diverse phytohormones and abiotic stress treatments (Figure 8). Among all evaluated genes, $B n G P X 21$ and BnGPX23 showed higher expression under almost all stress treatments except GA (Figure 8). These consequences agreed with earlier discoveries where several GPX genes exhibited sophisticated expression against stress treatments. For example, in watermelon, almost all ClGPX genes were up-regulated in response to cold, drought, and salinity stresses [54]. Nearly all T. salsuginea TsGPXs genes were suggestively up-regulated at a one-time point under salinity and drought treatments [56]. Pennisetum glaucum PgGPX expression levels were highly induced by salinity and drought conditions [12]. In cucumber, almost all six CsGPX genes were significantly up-regulated at some time points against cold, salinity, drought, and ABA treatments [53]. These discoveries offered robust evidence that GPX genes play a well-maintained role in defending abiotic and hormone stresses in diverse plant species.

\section{Conclusions}

In conclusion, a total of $25 B n G P X$ genes were identified in the rapeseed genome. To gain in-depth insights into the evolution of the GPX gene family in rapeseed genome, gene structure, phylogenetic and synteny, conserved motifs, cis-elements, GO annotation, miRNA prediction, tissue-specific expression, and expression profiling against diverse hormones, and abiotic stress treatments were carried out. The BnGPX genes were differentially expressed in numerous tissues/organs, signifying that these genes might contribute precise roles in rapeseed development. The outcomes of gene expression profiling in response to diverse abiotic stresses and hormones treatments discovered that several BnGPX genes might contribute to stress responses and hormone signaling pathways. These discoveries will lay the basis for studying the roles of $B n G P X$ genes in rapeseed developmental processes and response to several stresses using different functional validation options, such as overexpression, knockout via CRISPR/Cas system, etc.

Supplementary Materials: The following are available online at https: / www.mdpi.com/article / 10.3390/antiox10091481/s1, Table S1: A list of primers used for gene expression analysis in qRT-PCR, Table S2: The sequence of GPX family genes in Brassica napus, Arabidopsis thaliana, Brassica oleracea, and Brassica rapa, Table S3: The data of gene duplication type, $\mathrm{Ka}, \mathrm{Ks}$, and $\mathrm{Ka} / \mathrm{Ks}$ ratio values of $B$. napus and other three plant species, Table S4: Information on the 10 identified motifs in BnGPX proteins, Table S5: Information on hormone- and stress-related cis-elements identified in the promoters regions of BnGPXs, Table S6: Information on miRNA targeted BnGPX genes, Table S7: The GO enrichment analysis of $B n G P X$ genes.

Author Contributions: Conceptualization, W.L., X.H., P.L., A.R. and R.S.A.K.; methodology, W.L. and A.R.; software, W.L., X.H., P.L. and A.R.; validation, W.L., X.H., P.L. and A.R.; investigation, W.L., X.H., P.L. and A.R.; writing—original draft preparation, W.L., X.H., P.L. and A.R.; writing-review and editing, M.S.M., B.Z., J.P., S.F. and M.H.; supervision, W.L., X.H., P.L., A.R. and R.S.A.K. All authors have read and agreed to the published version of the manuscript.

Funding: This research received no external funding.

Institutional Review Board Statement: Not applicable.

Informed Consent Statement: Not applicable.

Data Availability Statement: The datasets used and/or analyzed during the current study are shown in the supplementary files. For tissue-specific expression profiling, we downloaded RNA- 
seq data of rapeseed (BioProject ID: PRJCA001495, accessed on 1 July 2021) from the National Genomics Data Center. The rapeseed genome sequence was downloaded from BnPIR database (http: / / cbi.hzau.edu.cn/bnapus/index.php, accessed on 1 July 2021). The sequences of Brassica rapa and Brassica oleracea are available at Phytozome database (https: / phytozome.jgi.doe.gov/pz/portal.html, accessed on 1 July 2021).

Conflicts of Interest: The authors declare no conflict of interest.

\section{References}

1. Raza, A.; Ashraf, F.; Zou, X.; Zhang, X.; Tosif, H. Plant Adaptation and Tolerance to Environmental Stresses: Mechanisms and Perspectives. In Plant Ecophysiology and Adaptation under Climate Change: Mechanisms and Perspectives I; Springer: Singapore, 2020; pp. 117-145.

2. Saddiq, M.S.; Afzal, I.; Iqbal, S.; Hafeez, M.B.; Raza, A. Low leaf sodium content improves the grain yield and physiological performance of wheat genotypes in saline-sodic soil. Pesqui. Agropecuária Trop. 2021, 51, e67663. [CrossRef]

3. Sharif, R.; Raza, A.; Chen, P.; Li, Y.; El-Ballat, E.M.; Rauf, A.; Hano, C.; El-Esawi, M.A. HD-ZIP Gene Family: Potential Roles in Improving Plant Growth and Regulating Stress-Responsive Mechanisms in Plants. Genes 2021, 12, 1256. [CrossRef] [PubMed]

4. Zafar, S.A.; Zaidi, S.S.-E.-A.; Gaba, Y.; Singla-Pareek, S.L.; Dhankher, O.P.; Li, X.; Mansoor, S.; Pareek, A. Engineering abiotic stress tolerance via CRISPR/Cas-mediated genome editing. J. Exp. Bot. 2020, 71, 470-479. [CrossRef]

5. Hasanuzzaman, M.; Bhuyan, M.; Zulfiqar, F.; Raza, A.; Mohsin, S.M.; Mahmud, J.A.; Fujita, M.; Fotopoulos, V. Reactive Oxygen Species and Antioxidant Defense in Plants under Abiotic Stress: Revisiting the Crucial Role of a Universal Defense Regulator. Antioxidants 2020, 9, 681. [CrossRef] [PubMed]

6. Mittler, R. ROS are good. Trends Plant Sci. 2017, 22, 11-19. [CrossRef]

7. Zafar, S.A.; Hameed, A.; Ashraf, M.; Khan, A.S.; Li, X.; Siddique, K.H. Agronomic, physiological and molecular characterisation of rice mutants revealed the key role of reactive oxygen species and catalase in high-temperature stress tolerance. Funct. Plant Biol. 2020, 47, 440-453. [CrossRef] [PubMed]

8. Ahmed, S.; Rashid, M.A.R.; Zafar, S.A.; Azhar, M.T.; Waqas, M.; Uzair, M.; Rana, I.A.; Azeem, F.; Chung, G.; Ali, Z. Genomewide investigation and expression analysis of APETALA-2 transcription factor subfamily reveals its evolution, expansion and regulatory role in abiotic stress responses in Indica Rice (Oryza sativa L. ssp. indica). Genomics 2021, 113, 1029-1043. [CrossRef] [PubMed]

9. Bela, K.; Horváth, E.; Gallé, Á.; Szabados, L.; Tari, I.; Csiszár, J. Plant glutathione peroxidases: Emerging role of the antioxidant enzymes in plant development and stress responses. J. Plant Physiol. 2015, 176, 192-201. [CrossRef] [PubMed]

10. Navrot, N.; Collin, V.; Gualberto, J.; Gelhaye, E.; Hirasawa, M.; Rey, P.; Knaff, D.B.; Issakidis, E.; Jacquot, J.-P.; Rouhier, N. Plant glutathione peroxidases are functional peroxiredoxins distributed in several subcellular compartments and regulated during biotic and abiotic stresses. Plant Physiol. 2006, 142, 1364-1379. [CrossRef]

11. Margis, R.; Dunand, C.; Teixeira, F.K.; Margis-Pinheiro, M. Glutathione peroxidase family-an evolutionary overview. FEBS J. 2008, 275, 3959-3970. [CrossRef]

12. Islam, T.; Manna, M.; Reddy, M.K. Glutathione peroxidase of Pennisetum glaucum $(P g G P x)$ is a functional Cd ${ }^{2+}$ dependent peroxiredoxin that enhances tolerance against salinity and drought stress. PLoS ONE 2015, 10, e0143344. [CrossRef] [PubMed]

13. Nareshkumar, A.; Subbarao, S.; Vennapusa, A.R.; Ashwin, V.; Banarjee, R.; Kulkarni, M.J.; Ramu, V.S.; Udayakumar, M. Enzymatic and non-enzymatic detoxification of reactive carbonyl compounds improves the oxidative stress tolerance in cucumber, tobacco and rice seedlings. J. Plant Growth Regul. 2020, 39, 1359-1372. [CrossRef]

14. Pour-Aboughadareh, A.; Omidi, M.; Naghavi, M.R.; Etminan, A.; Mehrabi, A.A.; Poczai, P. Wild relatives of wheat respond well to water deficit stress: A comparative study of antioxidant enzyme activities and their encoding gene expression. Agriculture 2020, 10, 415. [CrossRef]

15. Sreelakshmy, V.; Anbarasi, G.; Vishnupriya, B. Salicylic acid pre-treatment induced physiological and biochemical changes in Solanum lycopersicum L. under salinity stress. Not. Sci. Biol. 2021, 13, 10917. [CrossRef]

16. Heidari, P.; Entazari, M.; Ebrahimi, A.; Ahmadizadeh, M.; Vannozzi, A.; Palumbo, F.; Barcaccia, G. Exogenous EBR Ameliorates Endogenous Hormone Contents in Tomato Species under Low-Temperature Stress. Horticulturae 2021, 7, 84. [CrossRef]

17. Amarasekara, I.; Withanage, S.; Palihakkara, I. Quantitative gene expression analysis of selected genes to screen drought tolerance of selected Hevea Clones. Int. J. Res. Appl. Sci. Biotechnol. 2020, 7, 46-53. [CrossRef]

18. Navabpour, S.; Yamchi, A.; Bagherikia, S.; Kafi, H. Lead-induced oxidative stress and role of antioxidant defense in wheat (Triticum aestivum L.). Physiol. Mol. Biol. Plants 2020, 26, 793-802. [CrossRef]

19. Akbudak, M.A.; Filiz, E.; Vatansever, R.; Kontbay, K. Genome-wide identification and expression profiling of ascorbate peroxidase (APX) and glutathione peroxidase (GPX) genes under drought stress in sorghum (Sorghum bicolor L.). J. Plant Growth Regul. 2018, 37, 925-936. [CrossRef]

20. Lima-Melo, Y.; Carvalho, F.E.; Martins, M.O.; Passaia, G.; Sousa, R.H.; Neto, M.C.L.; Margis-Pinheiro, M.; Silveira, J.A. Mitochondrial GPX1 silencing triggers differential photosynthesis impairment in response to salinity in rice plants. J. Integr. Plant Biol. 2016, 58, 737-748. [CrossRef]

21. Passaia, G.; Caverzan, A.; Fonini, L.; Carvalho, F.; Silveira, J.; Margis-Pinheiro, M. Chloroplastic and mitochondrial GPX genes play a critical role in rice development. Biol. Plant. 2014, 58, 375-378. [CrossRef] 
22. Zhang, L.; Wu, M.; Teng, Y.; Jia, S.; Yu, D.; Wei, T.; Chen, C.; Song, W. Overexpression of the glutathione peroxidase 5 (RcGPX5) gene from rhodiola crenulata increases drought tolerance in Salvia miltiorrhiza. Front. Plant Sci. 2019, 9, 1950. [CrossRef] [PubMed]

23. Riyazuddin, R.; Bela, K.; Horváth, E.; Rigó, G.; Gallé, Á.; Szabados, L.; Fehér, A.; Csiszár, J. Overexpression of the Arabidopsis glutathione peroxidase-like 5 gene (AtGPXL5) resulted in altered plant development and redox status. Environ. Exp. Bot. 2019, 167, 103849. [CrossRef]

24. Raza, A. Eco-physiological and Biochemical Responses of Rapeseed (Brassica napus L.) to Abiotic Stresses: Consequences and Mitigation Strategies. J. Plant Growth Regul. 2021, 40, 1368-1388. [CrossRef]

25. He, H.; Lei, Y.; Yi, Z.; Raza, A.; Zeng, L.; Yan, L.; Xiaoyu, D.; Yong, C.; Xiling, Z. Study on the mechanism of exogenous serotonin improving cold tolerance of rapeseed (Brassica napus L.) seedlings. Plant Growth Regul. 2021, 94, 161-170. [CrossRef]

26. Raza, A.; Razzaq, A.; Mehmood, S.S.; Hussain, M.A.; Wei, S.; He, H.; Zaman, Q.U.; Xuekun, Z.; Yong, C.; Hasanuzzaman, M. Omics: The way forward to enhance abiotic stress tolerance in Brassica napus L. GM Crop. Food 2021, 12, 251-281. [CrossRef]

27. Su, W.; Raza, A.; Zeng, L.; Gao, A.; Lv, Y.; Ding, X.; Cheng, Y.; Zou, X. Genome-wide analysis and expression patterns of lipid phospholipid phospholipase gene family in Brassica napus L. BMC Genom. 2021, 22, 548. [CrossRef]

28. Raza, A.; Su, W.; Gao, A.; Mehmood, S.S.; Hussain, M.A.; Nie, W.; Lv, Y.; Zou, X.; Zhang, X. Catalase (CAT) Gene Family in Rapeseed (Brassica napus L.): Genome-Wide Analysis, Identification, and Expression Pattern in Response to Multiple Hormones and Abiotic Stress Conditions. Int. J. Mol. Sci. 2021, 22, 4281. [CrossRef]

29. Su, W.; Raza, A.; Gao, A.; Jia, Z.; Zhang, Y.; Hussain, M.A.; Mehmood, S.S.; Cheng, Y.; Lv, Y.; Zou, X. Genome-Wide Analysis and Expression Profile of Superoxide Dismutase (SOD) Gene Family in Rapeseed (Brassica napus L.) under Different Hormones and Abiotic Stress Conditions. Antioxidants 2021, 10, 1182. [CrossRef]

30. Song, J.M.; Liu, D.X.; Xie, W.Z.; Yang, Z.; Guo, L.; Liu, K.; Yang, Q.Y.; Chen, L.L. BnPIR: Brassica napus Pan-genome Information Resource for 1689 accessions. Plant Biotechnol. J. 2021, 19, 412. [CrossRef]

31. Rhee, S.Y.; Beavis, W.; Berardini, T.Z.; Chen, G.; Dixon, D.; Doyle, A.; Garcia-Hernandez, M.; Huala, E.; Lander, G.; Montoya, M. The Arabidopsis Information Resource (TAIR): A model organism database providing a centralized, curated gateway to Arabidopsis biology, research materials and community. Nucleic Acids Res. 2003, 31, 224-228. [CrossRef] [PubMed]

32. Finn, R.D.; Clements, J.; Arndt, W.; Miller, B.L.; Wheeler, T.J.; Schreiber, F.; Bateman, A.; Eddy, S.R. HMMER web server: 2015 update. Nucleic Acids Res. 2015, 43, W30-W38. [CrossRef]

33. Goodstein, D.M.; Shu, S.; Howson, R.; Neupane, R.; Hayes, R.D.; Fazo, J.; Mitros, T.; Dirks, W.; Hellsten, U.; Putnam, N. Phytozome: A comparative platform for green plant genomics. Nucleic Acids Res. 2012, 40, D1178-D1186. [CrossRef]

34. Gasteiger, E.; Hoogland, C.; Gattiker, A.; Wilkins, M.R.; Appel, R.D.; Bairoch, A. Protein identification and analysis tools on the ExPASy server. In The Proteomics Protocols Handbook; Humana Press Inc.: Totowa, NJ, USA, 2005; pp. 571-607.

35. Horton, P.; Park, K.-J.; Obayashi, T.; Fujita, N.; Harada, H.; Adams-Collier, C.; Nakai, K. WoLF PSORT: Protein localization predictor. Nucleic Acids Res. 2007, 35, W585-W587. [CrossRef]

36. Chen, C.; Chen, H.; Zhang, Y.; Thomas, H.R.; Frank, M.H.; He, Y.; Xia, R. TBtools: An integrative toolkit developed for interactive analyses of big biological data. Mol. Plant 2020, 13, 1194-1202. [CrossRef]

37. Bailey, T.L.; Boden, M.; Buske, F.A.; Frith, M.; Grant, C.E.; Clementi, L.; Ren, J.; Li, W.W.; Noble, W.S. MEME SUITE: Tools for motif discovery and searching. Nucleic Acids Res. 2009, 37, W202-W208. [CrossRef]

38. Kumar, S.; Stecher, G.; Li, M.; Knyaz, C.; Tamura, K. MEGA X: Molecular evolutionary genetics analysis across computing platforms. Mol. Biol. Evol. 2018, 35, 1547-1549. [CrossRef]

39. Subramanian, B.; Gao, S.; Lercher, M.J.; Hu, S.; Chen, W.-H. Evolview v3: A webserver for visualization, annotation, and management of phylogenetic trees. Nucleic Acids Res. 2019, 47, W270-W275. [CrossRef] [PubMed]

40. Tang, H.; Bowers, J.E.; Wang, X.; Ming, R.; Alam, M.; Paterson, A.H. Synteny and collinearity in plant genomes. Science 2008, 320, 486-488. [CrossRef] [PubMed]

41. Wang, D.; Zhang, Y.; Zhang, Z.; Zhu, J.; Yu, J. KaKs_Calculator 2.0: A toolkit incorporating gamma-series methods and sliding window strategies. Genom. Proteom. Bioinform. 2010, 8, 77-80. [CrossRef]

42. Lescot, M.; Déhais, P.; Thijs, G.; Marchal, K.; Moreau, Y.; Van de Peer, Y.; Rouzé, P.; Rombauts, S. PlantCARE, a database of plant cis-acting regulatory elements and a portal to tools for in silico analysis of promoter sequences. Nucleic Acids Res. 2002, 30, 325-327. [CrossRef]

43. Dai, X.; Zhuang, Z.; Zhao, P.X. psRNATarget: A plant small RNA target analysis server (2017 release). Nucleic Acids Res. 2018, 46, W49-W54. [CrossRef]

44. Powell, S.; Forslund, K.; Szklarczyk, D.; Trachana, K.; Roth, A.; Huerta-Cepas, J.; Gabaldon, T.; Rattei, T.; Creevey, C.; Kuhn, M. eggNOG v4. 0: Nested orthology inference across 3686 organisms. Nucleic Acids Res. 2014, 42, D231-D239. [CrossRef]

45. Swift, M.L. GraphPad prism, data analysis, and scientific graphing. J. Chem. Inf. Comput. Sci. 1997, 37, 411-412. [CrossRef]

46. Wang, Z.; Chen, Y.; Fang, H.; Shi, H.; Chen, K.; Zhang, Z.; Tan, X. Selection of reference genes for quantitative reverse-transcription polymerase chain reaction normalization in Brassica napus under various stress conditions. Mol. Genet. Genom. 2014, 289, 10231035. [CrossRef]

47. Cannon, S.B.; Mitra, A.; Baumgarten, A.; Young, N.D.; May, G. The roles of segmental and tandem gene duplication in the evolution of large gene families in Arabidopsis thaliana. BMC Plant Biol. 2004, 4, 10. [CrossRef]

48. Hurst, L.D. The Ka/Ks ratio: Diagnosing the form of sequence evolution. Trends Genet. TIG 2002, 18, 486. [CrossRef] 
49. Song, J.-M.; Guan, Z.; Hu, J.; Guo, C.; Yang, Z.; Wang, S.; Liu, D.; Wang, B.; Lu, S.; Zhou, R. Eight high-quality genomes reveal pan-genome architecture and ecotype differentiation of Brassica napus. Nat. Plants 2020, 6, 34-45. [CrossRef] [PubMed]

50. Mehmood, S.S.; Lu, G.; Luo, D.; Hussain, M.A.; Raza, A.; Zafar, Z.; Zhang, X.; Cheng, Y.; Zou, X.; Lv, Y. Integrated Analysis of Transcriptomics and Proteomics provides insights into the molecular regulation of cold response in Brassica napus. Environ. Exp. Bot. 2021, 187, 104480. [CrossRef]

51. Jana, G.A.; Yaish, M.W. Genome-wide identification and functional characterization of glutathione peroxidase genes in date palm (Phoenix dactylifera L.) under stress conditions. Plant Gene 2020, 23, 100237. [CrossRef]

52. Zhang, L.; Wu, M.; Yu, D.; Teng, Y.; Wei, T.; Chen, C.; Song, W. Identification of glutathione peroxidase (GPX) gene family in rhodiola crenulata and gene expression analysis under stress conditions. Int. J. Mol. Sci. 2018, 19, 3329. [CrossRef] [PubMed]

53. Zhou, Y.; Hu, L.; Ye, S.; Jiang, L.; Liu, S. Genome-wide identification of glutathione peroxidase (GPX) gene family and their response to abiotic stress in cucumber. 3 Biotech 2018, 8, 159. [CrossRef] [PubMed]

54. Zhou, Y.; Li, J.; Wang, J.; Yang, W.; Yang, Y. Identification and characterization of the glutathione peroxidase (GPX) gene family in watermelon and its expression under various abiotic stresses. Agronomy 2018, 8, 206. [CrossRef]

55. Ramos, J.; Matamoros, M.A.; Naya, L.; James, E.K.; Rouhier, N.; Sato, S.; Tabata, S.; Becana, M. The glutathione peroxidase gene family of Lotus japonicus: Characterization of genomic clones, expression analyses and immunolocalization in legumes. New Phytol. 2009, 181, 103-114. [CrossRef] [PubMed]

56. Gao, F.; Chen, J.; Ma, T.; Li, H.; Wang, N.; Li, Z.; Zhang, Z.; Zhou, Y. The glutathione peroxidase gene family in Thellungiella salsuginea: Genome-wide identification, classification, and gene and protein expression analysis under stress conditions. Int. J. Mol. Sci. 2014, 15, 3319-3335. [CrossRef] [PubMed]

57. Huan, C.; Jiang, L.; An, X.; Kang, R.; Yu, M.; Ma, R.; Yu, Z. Potential role of glutathione peroxidase gene family in peach fruit ripening under combined postharvest treatment with heat and 1-MCP. Postharvest Biol. Technol. 2016, 111, 175-184. [CrossRef]

58. Chen, M.; Li, K.; Li, H.; Song, C.-P.; Miao, Y. The glutathione peroxidase gene family in gossypium hirsutum: Genome-wide identification, classification, gene expression and functional analysis. Sci. Rep. 2017, 7, 44743. [CrossRef]

59. Tyagi, S.; Sembi, J.K.; Upadhyay, S.K. Gene architecture and expression analyses provide insights into the role of glutathione peroxidases (GPXs) in bread wheat (Triticum aestivum L.). J. Plant Physiol. 2018, 223, 19-31. [CrossRef] [PubMed]

60. Xu, G.; Guo, C.; Shan, H.; Kong, H. Divergence of duplicate genes in exon-intron structure. Proc. Natl. Acad. Sci. USA 2012, 109, 1187-1192. [CrossRef]

61. Maruyama-Nakashita, A.; Nakamura, Y.; Watanabe-Takahashi, A.; Inoue, E.; Yamaya, T.; Takahashi, H. Identification of a novel cis-acting element conferring sulfur deficiency response in Arabidopsis roots. Plant J. 2005, 42, 305-314. [CrossRef]

62. Chen, L.; Chen, L.; Zhang, X.; Liu, T.; Niu, S.; Wen, J.; Yi, B.; Ma, C.; Tu, J.; Fu, T. Identification of miRNAs that regulate silique development in Brassica napus. Plant Sci. 2018, 269, 106-117. [CrossRef] [PubMed]

63. Buhtz, A.; Springer, F.; Chappell, L.; Baulcombe, D.C.; Kehr, J. Identification and characterization of small RNAs from the phloem of Brassica napus. Plant J. 2008, 53, 739-749. [CrossRef] [PubMed]

64. Fu, Y.; Mason, A.S.; Zhang, Y.; Lin, B.; Xiao, M.; Fu, D.; Yu, H. MicroRNA-mRNA expression profiles and their potential role in cadmium stress response in Brassica napus. BMC Plant Biol. 2019, 19, 570. [CrossRef] [PubMed]

65. Körbes, A.P.; Machado, R.D.; Guzman, F.; Almerao, M.P.; de Oliveira, L.F.V.; Loss-Morais, G.; Turchetto-Zolet, A.C.; Cagliari, A.; dos Santos Maraschin, F.; Margis-Pinheiro, M. Identifying conserved and novel microRNAs in developing seeds of Brassica napus using deep sequencing. PLoS ONE 2012, 7, e50663.

66. Shen, E.; Zou, J.; Hubertus Behrens, F.; Chen, L.; Ye, C.; Dai, S.; Li, R.; Ni, M.; Jiang, X.; Qiu, J. Identification, evolution, and expression partitioning of miRNAs in allopolyploid Brassica napus. J. Exp. Bot. 2015, 66, 7241-7253. [CrossRef]

67. Feng, H.; Xu, M.; Gao, Y.; Liang, J.; Guo, F.; Guo, Y.; Huang, L. Vm-milR37 contributes to pathogenicity by regulating glutathione peroxidase gene VmGP in Valsa mali. Mol. Plant Pathol. 2021, 22, 243-254. [CrossRef]

68. Shan, T.; Fu, R.; Xie, Y.; Chen, Q.; Wang, Y.; Li, Z.; Song, X.; Li, P.; Wang, B. Regulatory mechanism of maize (Zea mays L.) miR164 in salt stress response. Russ. J. Genet. 2020, 56, 835-842. [CrossRef]

69. Fang, Y.; Xie, K.; Xiong, L. Conserved miR164-targeted NAC genes negatively regulate drought resistance in rice. J. Exp. Bot. 2014, 65, 2119-2135. [CrossRef]

70. Feng, H.; Duan, X.; Zhang, Q.; Li, X.; Wang, B.; Huang, L.; Wang, X.; Kang, Z. The target gene of tae-miR164, a novel NAC transcription factor from the NAM subfamily, negatively regulates resistance of wheat to stripe rust. Mol. Plant Pathol. 2014, 15, 284-296. [CrossRef]

71. Yuan, S.; Zhao, J.; Li, Z.; Hu, Q.; Yuan, N.; Zhou, M.; Xia, X.; Noorai, R.; Saski, C.; Li, S. MicroRNA396-mediated alteration in plant development and salinity stress response in creeping bentgrass. Hortic. Res. 2019, 6, 48. [CrossRef] [PubMed]

72. Yuan, S.; Li, Z.; Yuan, N.; Hu, Q.; Zhou, M.; Zhao, J.; Li, D.; Luo, H. MiR396 is involved in plant response to vernalization and flower development in Agrostis stolonifera. Hortic. Res. 2020, 7, 173. [CrossRef]

73. Gao, P.; Bai, X.; Yang, L.; Lv, D.; Li, Y.; Cai, H.; Ji, W.; Guo, D.; Zhu, Y. Over-expression of osa-MIR396c decreases salt and alkali stress tolerance. Planta 2010, 231, 991-1001. [CrossRef] [PubMed] 